\title{
Research Paper: Comparing Electromyographic Ac- tivity of Quadriceps Muscle During Straight Leg Raise in Individuals With and Without Patellofemoral Pain Syndrome
}

\author{
Hiva Lotfi ${ }^{1}$ (D), Afsun Nodehi Moghadam ${ }^{1 *}$ (D), Mohsen Shati ${ }^{2}$ \\ 1. Department of Physiotherapy, University of Social Welfare and Rehabilitation Sciences, Tehran, Iran. \\ 2. Department of Ageing, University of Social Welfare and Rehabilitation Sciences, Tehran, Iran.
}

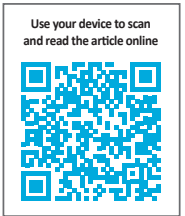

Citat on Lotfi H, Nodehi Moghadam A, Shati M. Comparing Electromyographic Activity of Quadriceps Muscle During Straight Leg Raise in Individuals With and Without Patellofemoral Pain Syndrome. Physical Treatments. 2018; 7(4):197-204. http://dx.doi.org/10.32598/ptj.7.4.197

dol: : http://dx.doi.org/10.32598/ptj.7.4.197

Funding: See Page 202

\section{(c) (i) (5)}

\section{Article info:}

Received: 26 Aug 2017

Accepted: 23 Nov 2017

Available Online: 01 Jan 2018

Keywords:

Vastus Medialis Obliquus, Vastus Lateralis, Straight Leg Raise, Patellafemoral Pain Syndrome, Electromyography

\section{A B S T RA C T}

Purpose: This study aimed to investigate the electromyographic activities and balance of Vastus Medialis Obliquus (VMO) and Vastus Lateralis (VL) muscles during Straight Leg Raise (SLR) in individuals with and without Patellofemoral Pain Syndrome (PFPS).

Methods: Through an analytical case-control study, 26 persons with PFPS and 26 healthy subjects were recruited by non-random and convenience sampling method. All subjects performed SLR movement. The EMG activity of VMO, VL and VMO/VL ratio were recorded and measured. The muscles activation level were compared between the 2 groups using the Independent $t$ test. The obtained data were analyzed using SPSS.

Results: The mean and maximum EMG activity of VMO, VL and VMO/VL ratio did not have any significant differences between the PFPS and healthy groups $(\mathrm{P}>0.05)$. In other words, during SLR, the VMO and VL muscles activation in the PFPS group were approximately the same as the healthy group.

Conclusion: These data suggest that not all individuals with PFPS had weak quadriceps muscles. Many factors may contribute to PFPS that should be considered in the assessment and rehabilitation of individuals with PFPS.

\footnotetext{
* Corresponding Author:

Afsun Nodehi Moghadam, PhD

Address: Department of Physiotherapy, University of Social Welfare and Rehabilitation Sciences, Tehran, Iran.

Phone: +98 (21) 22180084

E-mail:afsoonnodehi@gmail.com
} 
Highlights

- The EMG activity of vastus medialis obliquus and vastus lateralis muscles and of their ratio didn't have any significant differences between individuals with and without patellofemoral pain syndrome.

- The vastus medialis obliquus and vastus lateralis muscles activation in the patellofemoral pain syndrome group were approximately the same as the healthy group.

- All individuals with patellofemoral pain syndrome didn't have weak quadriceps muscle.

\section{Plain Language Summary}

This study aimed to investigate the activity of 2 parts of quadriceps muscle during Straight Leg Raise (SLR) in individuals with and without Patellofemoral Pain Syndrome (PFPS). A total of 26 persons with PFPS and 26 healthy subjects participated in this study. Therefore, the muscles activation levels were compared between the 2 groups. The results showed that during SLR, the activation of these 2 parts of quadriceps muscle in the PFPS group were approximately the same as the healthy group. Many factors may contribute to PFPS and all individuals with PFPS didn't have weak quadriceps muscles.

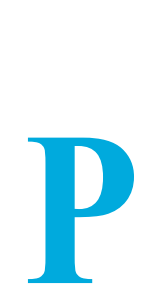

\section{Introduction}

atellofemoral Pain Syndrome (PFPS) is a frequent knee problem, mostly reported in females and athletes. Patients with PFPS experience anterior or retropatellar knee pain during movements such as running, jumping, climbing the stairs, or quad sitting for a long time [1-3].

Some factors including lower extremity malalignment, patella alta, lateral patellar tilt, increased Quadriceps (Q) angle, knee valgus, femoral anteversion, foot hyperpronation influence patellofemoral biomechanics and increase the risk for PFPS $[4,5]$. In addition, soft tissue imbalance such as lateral retinaculum shortening or medial retinaculum weakness, iliotibial band tightness, hamstring and gastrocnemius can indirectly increase the forces on the patellofemoral joint $[6,7]$. Quadriceps muscle (especially VMO and VL parts) plays a critical role in maintaining patella in the trochlea groove.

The Vastus Medialis Obliquus (VMO) is considered as the main active medial stabilizer of the patella, according to its fibers orientation. While, Vastus Lateralis muscle (VL), iliotibial band, and lateral retinaculum are lateral patellar stabilizers $[5,8]$. Therefore, weakness or delay of the VMO activity causes the imbalance between VMO and VL muscles. This can increase lateral force on the patella which is one of the causes of PFPS [9-11].
Conventional treatment usually includes quadriceps strengthening, patellar bracing and taping, and soft tissue mobilization and stretching [12]. In PFPS rehabilitation, quadriceps muscle strengthening, especially VMO part has been considered as an important intervention. Prior research suggests the importance of PFPS rehabilitation program on strengthening and correcting the timing of the VMO to improve the $\mathrm{VMO} / \mathrm{VL}$ ratio [13]. Also, optimal activity of the VMO, compared to the VL is important in improving the balance between the VMO and VL muscles and correcting biomechanics of the patella in trochlear groove that reduce the patellofemoral joint stress [14].

Balance between the VMO and VL muscles activation and improvement of $\mathrm{VMO} / \mathrm{VL}$ ratio is essential in individuals with PFPS. Previous studies investigated the effect of quadriceps muscles strengthening exercises (especially VMO muscle) on the improvement of VMO/ VL ratio. The types of exercises included concentric, eccentric, isotonic, isometric and isokinetic in closed chain or open chain conditions $[6,14,15]$. One of the recommended exercises in individuals with PFPS is Straight Leg Raise (SLR). The effect of additional hip rotation, dorsiflexion, or plantar flexion, and adduction exercises have been evaluated in various studies $[2,5,8,16]$. However, there is no consensus over the most effective exercise therapy in PFPS.

There has been much discussion on the VMO/VL ratio and the balance of activity between the VMO and the VL in healthy and PFPS subjects. Some studies showed that 
$\mathrm{VMO} / \mathrm{VL}$ ratio in individuals with PFPS is less than that in the healthy population $[17,18]$. However, other studies have reported no significant differences between the healthy and PFPS subjects in VMO/VL ratio during SLR and SLR with hip external rotation $[2,19]$.

There is limited and conflicting information to support the change in quadriceps muscles activation and their weakness in individual with PFPS [20]. Therefore, this study aimed to investigate the electromyographic activities and balance of VMO and VL muscles during SLR in individuals with and without PFPS.

\section{Materials and Methods}

\section{Subjects}

Through an analytical case-control study, 26 persons with PFPS and 26 healthy subjects (18 women and 8 men, in each group) were recruited by non-random and convenience sampling method. The inclusion criteria for PFPS were having anterior or retropatellar knee pain (assessed by Visual Analogue Scale [VAS] of less than 5) during at least 2 of the following activities: prolonged sitting, squatting, running, ascending or descending stairs, kneeling and jumping, and . The Participants aged between $18-30$ years $[2,21]$. The exclusion criteria were history of knee surgery or injury and/or patellar dislocation or subluxation $[11,21]$.

\section{Electromyography (EMG) assessment}

EMG data were collected for the VMO and VL muscles using MT8 EMG device manufactured by MIE in the UK. The $\mathrm{Ag} / \mathrm{AgCl}$ surface electrodes were positioned at an inter-electrode distance of $2 \mathrm{~cm}$. In the EMG signal processing, the sampling frequency was set to $1000 \mathrm{~Hz}$. We used a band-pass filter of 20 to $450 \mathrm{~Hz}$ to reduce external noise $[2,22]$. The raw EMG signal was smoothed with root mean square method.

Skin was shaved and cleaned with an alcohol wipe to minimize the impedance prior to the attachment of the electrodes. The VMO muscle electrodes were placed approximately $4 \mathrm{~cm}$ superior and medial to the superomedial border of the patella at a $50^{\circ}$ to $55^{\circ}$ to the reference line (the reference line, joining the anterosuperior iliac spine to the center of the patella). The VL electrode was placed 10 $\mathrm{cm}$ superior to the superolateral border of the patella at approximately $15^{\circ}$ to the reference line. The ground electrode was placed on the patella. To prevent signal perturbation, the electrodes were fixed with Velcro strap [8, 23].

\section{Study procedure}

The participants laid supine on the bed and raised the lower limb vertically with knee straightened up to $30^{\circ}$ elevation that determined using a goniometer and a designed device. To control the speed of movement, the subjects were trained to reach the lower limb to the determined target point in $5 \mathrm{~s}$. Also, in seated position, the Maximal Voluntary Isometric Contraction (MVIC) of the quadriceps muscle against manual resistance was measured to compute the normalized data for VMO and VL muscles.

\section{Statistical analysis}

All obtained data were analyzed using SPSS. The Kolmogorov-Smirnov test was performed to confirm the normal distribution of the data $(\mathrm{P}>0.05)$. Test-retest reliability of EMG measurement was assessed by IntraClass Correlation (ICC). The Independent $t$ test was used to compare the demographic characteristics and muscles activation level between the PFPS and healthy groups. $(\mathrm{P}<0.05$, indicating a significant difference).

\section{Results}

The demographic characteristics of the subjects are presented in Table 1. No significant differences were found between the study groups in respect of age, height, weight, or body mass index (Table 1). The Intra-class Correlation Coefficient (ICC) values for assessing test-retest reliability of EMG activity of VMO and VL during SLR movement was greater than 0.93 . Table 2 summarizes the mean and maximum EMG activity of VMO, VL and VMO/VL ratios during SLR movement. The mean and maximum EMG activity of VMO, VL and VMO/VL ratios were not significantly different between the PFPS and healthy groups ( $\mathrm{P}>0.05$ ) (Table 2) (Figures 1, 2, and 3).

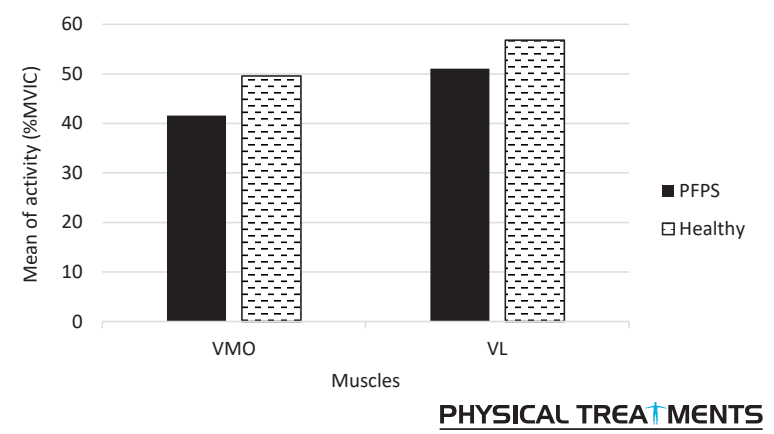

Figure 1. Mean activity of VMO and VL muscles in PFPS and healthy groups 
Table 1. Demographic characteristics of the subjects

\begin{tabular}{|c|c|c|c|}
\hline \multirow{3}{*}{ Characteristics } & \multicolumn{2}{|c|}{ Mean $\pm S D$} & \multirow{3}{*}{$\mathbf{P}$} \\
\hline & \multicolumn{2}{|c|}{$(n=26)$} & \\
\hline & PFPS & Healthy & \\
\hline Age, $y$ & $22.92 \pm 1.78$ & $23 \pm 1.91$ & 0.98 \\
\hline Height, $\mathrm{cm}$ & $168.81 \pm 8.52$ & $170 \pm 8.45$ & 0.61 \\
\hline Weight, kg & $65.73 \pm 11.47$ & $66.96 \pm 11.93$ & 0.70 \\
\hline $\mathrm{BMI}, \mathrm{kg} / \mathrm{m}^{2}$ & $22.97 \pm 2.21$ & $23.08 \pm 2.72$ & 0.88 \\
\hline Pain & $4.12 \pm 0.65$ & 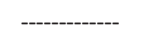 & \\
\hline
\end{tabular}

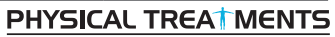

Table 2. Mean and maximum electromyography activity of VMO, VL and VMO/VL ratios during SLR between the PFPS and healthy groups (\%MVIC)

\begin{tabular}{|c|c|c|c|c|c|c|}
\hline \multirow{3}{*}{ Muscles Group } & \multicolumn{3}{|c|}{ Mean of Muscle Activity } & \multicolumn{3}{|c|}{ Maximum of Muscle Activity } \\
\hline & \multicolumn{2}{|c|}{ Mean $\pm S D$} & \multirow{2}{*}{$\mathbf{P}$} & \multicolumn{2}{|c|}{ Mean $\pm S D$} & \multirow{2}{*}{$\mathbf{P}$} \\
\hline & PFPS & Healthy & & PFPS & Healthy & \\
\hline VMO & $41.55 \pm 13.22$ & $49.57 \pm 19.70$ & 0.09 & $55.69 \pm 13.05$ & $59.74 \pm 18.40$ & 0.36 \\
\hline VL & $51.05 \pm 10.92$ & $56.78 \pm 15.84$ & 0.13 & $64.06 \pm 11.61$ & $64.35 \pm 16.57$ & 0.85 \\
\hline $\mathrm{VMO} / \mathrm{VL}$ & $0.81 \pm 0.18$ & $0.84 \pm 0.17$ & 0.58 & $0.86 \pm 0.15$ & $0.92 \pm 0.12$ & 0.13 \\
\hline
\end{tabular}

\section{Discussion}

Our results indicate that the mean and maximum EMG activity of $\mathrm{VMO}, \mathrm{VL}$ and $\mathrm{VMO} / \mathrm{VL}$ ratios lacked any significant differences between the individuals with and without PFPS. In this study, SLR activated VMO and VL for approximately $41 \%$ and $51 \%$ in the PFPS group and $50 \%$ and $57 \%$ in the healthy group, respectively. In addition, during SLR, the VMO/VL ratio was almost the same as the healthy group and there was no significant differences between the 2 groups. The VMO/VL ratio according to maximum EMG activity was close to the $1: 1$ ratio in the healthy group.

Unlike our study results, Souza et al. and Miller et al. reported that in individuals with PFPS, the VMO and VL muscles activity and VMO/VL ratio was less than the healthy individuals $[17,18]$. However, in agreement with our results, Choi et al. and Livecchi et al. reported no significant differences in the VMO and VL muscles

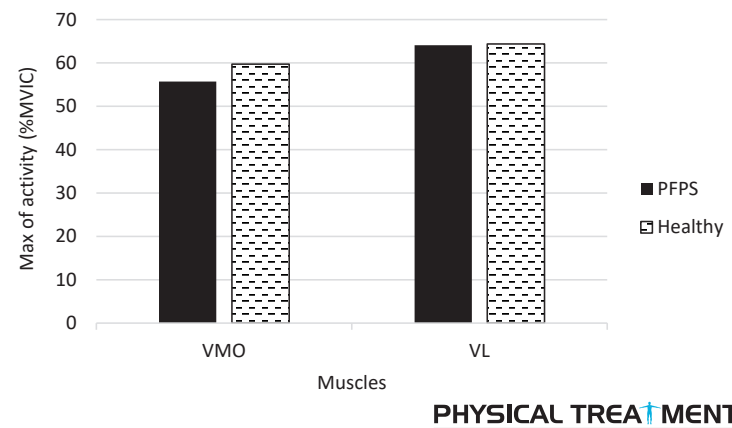

Figure 2. Maximum activity of VMOand VL muscles in PFPS and healthy group

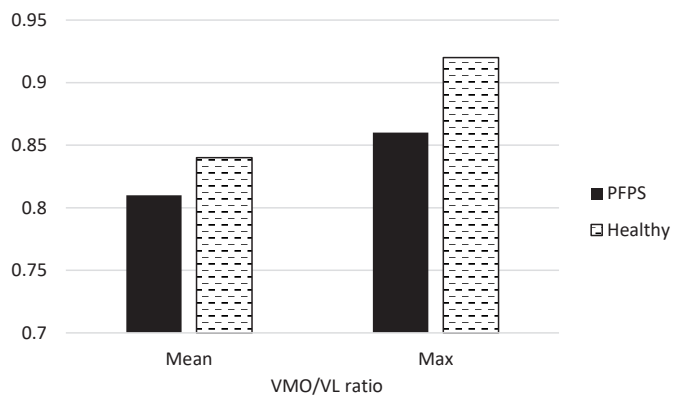

PHYSICAL TREA $\$ MENTS

Figure 3. VMO/VL ratio in PFPS and healthy groups 
activity and $\mathrm{VMO} / \mathrm{VL}$ ratio between the PFPS and healthy groups $[2,19]$. Moreover, Karst and Willett, and Powers et al. reported no significant differences in the timing or activation levels of the VMO and VL muscles in individuals with and without PFPS [24, 25]. They reported that quadriceps muscle onset time in subjects with and without PFPS was different during voluntary activity that might be a cause of PFPS [26-28].

A possible reason to the inconsistency in the results of studies lies in the level of pain in the PFPS subjects. In our study, PFPS subjects did not report much pain $(\mathrm{VAS}<5)$. It was shown that pain inhibits and decreases muscle activity, which also causes more muscle weakness [5]. It is difficult to determine whether quadriceps weakness or atrophy are predisposing factors or develop after the initiation of PFPS. Furthermore, longitudinal research is required to determine whether quadriceps weakness or atrophy accounts as predisposing factors for PFPS.

Quadriceps muscle strengthening exercises have been recommended as an effective intervention to decrease pain and improve function in individuals with PFPS. Pain free quadriceps exercise is a challenge for clinicians for safe treatment of PFPS. SLR is a common exercise prescribed for patients with knee disorders to strengthen the quadriceps muscle $[16,20]$, because exercising the knee in a fully extended position can facilitate quadriceps activation and reduce patellofemoral joint reaction forces [29-31]. Several studies assessed the quadriceps muscles activations during different types of SLR. Bolgla et al. [30] reported that during SLR, vastus medialis muscle had greater activation than 2 weight bearing exercises, including single leg stance and bilateral squat. However, in this study only healthy subjects participated and EMG activity of VL muscle was not measured.

Bakhtiary and Fatemi reported that semi-squat exercises increased the MIVC force of the quadriceps more than SLR [14]. However, they investigated quadriceps muscle activity as a whole and disregarded different parts of quadriceps separately, and lacked a control group. Moreover, Soderberg et al. reported higher activation of Rectus Femoris (RF) than vasti muscles during SLR [1, 32]. Sykes and Wong reported that additional hip rotation, especially external rotation to SLR had a significant effect on the EMG amplitude of VMO [33]. However, Karst and Jewett and Livecchi et al. stated that different hip position in SLR could not change the VMO activity $[19,34]$. Wong reported that additional ankle weight to SLR could improve the VMO muscle activity [35].
According the results of this study and previous studies, there is no agreement on muscles weakness or decrease in VMO/VL ratio [36]. Some studies reported muscles weakness and decrease in VMO/VL ratio [17, 18], while others reported no significant associations in this regard [1, 2, 19, 34]. Additionally, the effectiveness of exercises for the efficient activity of VMO muscle and improvement of VMO/VL ratio still remains unclear.

According to the previous studies, many factors may contribute to PFPS. PFPS is a multifactorial disease and various factors may be predisposition for the development of PFPS via alterations in patellar tracking, increased patellofemoral joint forces, or combinations of these bio-mechanical features [3]. This finding that not all people with PFPS have quadriceps weakness may explain why some individuals with PFPS respond better to quadriceps strengthening than others. In addition, recent studies reported weakness of the gluteus medius, gluteus maximus, and hip external rotators and poor proprioceptive control in patients with PFPS [6, 36]. Moreover, some studies indicated that combined interventions in patients with PFPS may result in better improvement of pain and function. Thus, PFPS is a multifactorial condition that one specific treatment may be beneficial for some patients. However, there is no evidence that one specific treatment could be effective for all patients. Thus, several factors may contribute to PFPS that should be considered in the assessment and rehabilitation of individuals with PFPS.

The mean and maximum EMG activity of $\mathrm{VMO}$, $\mathrm{VL}$, and VMO/VL ratios lacked significant differences between individuals with and without PFPS. In other words, during SLR the VMO and VL muscles activation ratios in the PFPS group were approximately the same as the healthy group. These obtained data suggest that not all individuals with PFPS had quadriceps weakness. Many factors may contribute to PFPS that should be considered in the assessment and rehabilitation of individuals with PFPS.

\section{Ethical Considerations}

\section{Compliance with ethical guidelines}

All participants signed an informed consent form to participate in this study that was approved by the Ethics Committee of University of Social Welfare and Rehabilitation Sciences (Code: IR.USWR.REC.1396.128). 


\section{Funding}

This research did not receive any specific grant from funding agencies in the public, commercial, or not-forprofit sectors.

\section{Conflict of interest}

The authors certify that they have no affiliation with or involvement in any organization or entity with any financial interest, or non- financial interest in the subject matter or materials dismissed in this manuscript.

\section{Acknowledgements}

The authors would like to thank the authorities in Department of Physiotherapy, University of Social Welfare and Rehabilitation Sciences.

\section{References}

[1] Kushion D, Rheaume J, Kopchitz K, Glass S, Alderink G, Jinn JH EMG activation of the vastus medialis oblique and vastus lateralis during four rehabilitative exercises. The Open Rehabilitation Journal. 2012; 5, 1-7. [DOI:10.2174/1874943701205010001]

[2] Choi SA, Cynn HS, Yoon TL, Choi WJ, Lee JH. Effects of ankle dorsiflexion on vastus medialis oblique and vastus lat eralis muscle activity during straight leg raise exercise with hip external rotation in patellofemoral pain syndrome. Journal of Musculoskeletal Pain. 2014; 22(3):260-7. [DOI:10.3109 /10582452.2014.907857

[3] Hryvniak D, Magrum E, Wilder R. Patellofemoral pain syndrome: An update. Current Physical Medicine and Rehabilitation Re-ports. 2014; 2(1):16-24. [DOI:10.1007/s40141-014-0044-3]

[4] Al-Hakim W, Jaiswal PK, Khan W, Johnstone D. The non-operative treatment of anterior knee pain. The Open Orthopaedics Journal. 2012; 6:320-6. [DOI:10.2174/1874325001206010320]

[5] Coqueiro KRR, Bevilaqua-Grossi D, Bérzin F, Soares AB, Candolo C, Monteiro-Pedro V. Analysis on the activa-tion of the VMO and VLL muscles during semisquat exercises with and without hip adduction in individuals with patellofemoral pain syndrome. Journal of Electromyography and Kinesiology. 2005; 15(6):596-603. [DOI:10.1016/j.jelee kin.2005.03.001]

[6] Dutton RA, Khadavi MJ, Fredericson M. Update on rehabilitation of patellofemoral pain. Current Sports Medi-cine Reports. 2014; 13(3):172-8. [DOI:10.1249/ JSR.00000000000000056]

[7] Saltychev M, Dutton RA, Laimi K, Beaupré GS, Virolainen P, Fredericson M. Effectiveness of conservative treatment for patellofemo-ral pain syndrome: A systematic review controlled study and meta-analysis. Journal of Rehabilitation Medicine. 2018; 50(5):393-401. [DOI:10.2340/16501977-2295]
[8] Belli G, Vitali L, Botteghi M, Vittori LN, Petracci E, Maietta Latessa P. Electromyographic analysis of leg exten-sion exercise during different ankle and knee positions. Journal of Mechanics in Medicine and Biology. 2015; 15(02):1540037. [DOI:10.1142/S0219519415400370]

[9] Pal S, Draper CE, Fredericson M, Gold GE, Delp SL, Beaupre GS, et al. Patellar maltracking correlates with vastus medialis activation delay in patellofemoral pain patients. The American Journal of Sports Medicine. 2011; 39(3):590-8 [DOI:10.1177/0363546510384233]

[10] Tang SF, Chen CK, Hsu R, Chou SW, Hong WH, Lew HL. Vastus medialis obliquus and vastus lateralis activity in open and closed kinetic chain exercises in patients with patellofemoral pain syndrome: an electromyographic study. Archives of Physical Medicine and Rehabilitation. 2001; 82(10):1441-5. [DOI:10.1053/apmr.2001.26252]

[11] Chang WD, Huang WS, Lee CL, Lin HY, Lai PT. Effects of open and closed kinetic chains of sling exercise ther-apy on the muscle activity of the vastus medialis oblique and vastus lateralis. Journal of Physical Therapy Sci-ence. 2014; 26(9):1363-6. [DOI:10.1589/jpts.26.1363]

[12] McConnell J, Bennell K. Conservative management of anterior knee pain: The McConnell program. In: Sanchis-Alfonso $\mathrm{V}$, editor. Anterior Knee Pain and Patellar Instability. London: Springer; 2011. [DOI:10.1007/978-0-85729-507-1_13]

[13] Kooiker L, Van De Port IG, Weir A, Moen MH. Effects of Physical therapist-guided quadriceps-strengthening exercises for the treat-ment of Patellofemoral Pain Syndrome: A systematic review. Journal of Orthopaedic \& Sports Physical Therapy. 2014; 44(6):391-B1. [DOI:10.2519/jospt.2014.4127]

[14] Bakhtiary AH, Fatemi E. Open versus closed kinetic chain exercises for patellar chondromalacia. British Journal of Sports Medicine. 2007; 42(2):99-102. [DOI:10.1136/ bjsm.2007.038109]

[15] Machado W, Paz G, Mendes L, Maia M, Winchester JB, Lima $\mathrm{V}$, et al. myoeletric activity of the quadriceps during leg press exercise performed with differing techniques. The Journal of Strength \& Conditioning Research. 2017 31(2):422-9. [PMID]

[16] Mikaili S, Khademi-Kalantari K, Rezasoltani A, Arzani P, Baghban AA. Quadriceps force production during straight leg raising at different hip positions with and without concomitant ankle dorsiflexion. Journal of Body-work and Movement Therapies. 2018; 22(4):904-8. [DOI:10.1016/j. jbmt.2017.11.006]

[17] Souza DR, Gross MT. Comparison of vastus medialis obliquus: Vastus lateralis muscle integrated electromyographic ratios between healthy subjects and patients with patellofemoral pain. Physical Therapy. 1991; 71(4):310-6. [DOI:10.1093/ptj/71.4.310]

[18] Miller JP, Sedory D, Croce RV. Vastus medialis obliquus and vastus lateralis activity in patients with and with-out patellofemoral pain syndrome. Journal of Sport Rehabilitation. 1997; 6(1):1-10. [DOI:10.1123/jsr.6.1.1]

[19] Livecchi NM, Armstrong CW, Cordova ML, Merrick MA Rankin JM. Vastus lateralis and vastus medialis obliquus activity during a straight-leg raise and knee extension with lateral hip rotation. Journal of Sport Reha-bilitation. 2002; 11(2):120-6. [DOI:10.1123/jsr.11.2.120] 
[20] O'sullivan SP, Popelas CA. Activation of vastus medialis obliquus among individuals with patellofemoral pain syndrome. Journal of Strength and Conditioning Research. 2005; 19(2):302-4. [DOI:10.1519/00124278-200505000-00011]

[21] Syme G, Rowe P, Martin D, Daly G. Disability in patients with chronic patellofemoral pain syndrome: A ran-domised controlled trial of $\mathrm{VMO}$ selective training versus general quadriceps strengthening. Manual Therapy. 2009; 14(3):25263. [DOI:10.1016/j.math.2008.02.007]

[22] Williams MR. Electromyographic analysis of hip and knee exercises: A continuum from early rehabilitation to enhancing performance [MSc. thesis]. Hatfield: University of Hertfordshire; 2014.

[23] Bevilaqua-Grossi D, Monteiro-Pedro V, Sousa G, Silva Z, Bérzin F. Contribution to the anatomical study of the oblique portion of the vastus lateralis muscle. Brazilian Journal of Morphological Sciences. 2004; 21(1):47-52.

[24] Karst GM, Willett GM. Onset timing of electromyographic activity in the vastus medialis oblique and vastus lat-eralis muscles in subjects with and without patellofemoral pain syndrome. Physical Therapy. 1995; 75(9):813-23. [DOI:10.1093/ptj/75.9.813]

[25] Powers CM, Landel R, Perry J. Timing and intensity of vastus muscle activity during functional activities in sub-jects with and without patellofemoral pain. Physical Therapy. 1996; 76(9):946-55. [DOI:10.1093/ptj/76.9.946]

[26] Voight ML, Wieder DL. Comparative reflex response times of vastus medialis obliquus and vastus lateralis in normal subjects and subjects with extensor mechanism dysfunction: An electromyographic study. The Ameri-can Journal of Sports Medicine. 1991; 19(2):131-7. [DOI:10.1177/036354 659101900207]

[27] Shelton G. Conservative management of patellofemoral dysfunction. Primary Care. 1992; 19(2):331-50. [PMID]

[28] Kannus P, NiittymÄki S. Which factors predict outcome in the nonoperative treatment of patellofemoral pain syndrome? A prospective follow-up study. Medicine \& Science in Sports \& Exercise. 1994; 26(3):289-96. [DOI:10.1249/00005768-199403000-00004]

[29] McMullen W, Roncarati A, Koval P. Static and isokinetic treatments of chondromalacia patella: A comparative investigation. Journal of Orthopaedic \& Sports Physical Therapy. 1990; 12(6):256-66. [DOI:10.2519/jospt.1990.12.6.256]

[30] Bolgla LA, Shaffer SW, Malone TR. Vastus medialis activation during knee extension exercises: Evidence for ex-ercise prescription. Journal of Sport Rehabilitation. 2008; 17(1):110. [DOI:10.1123/jsr.17.1.1]

[31] Greenfield BH. Rehabilitation of the Knee: A Problem Solving Approach. Philadelphia, Pennsylvania: F.A. Davis Company; 1993.

[32] Soderberg GL, Cook TM. An electromyographic analysis of quadriceps femoris muscle setting and straight leg raising. Physical Therapy. 1983; 63(9):1434-8. [DOI:10.1093/ $\mathrm{ptj} / 63.9 .1434]$

[33] Sykes K, Wong YM. Electrical activity of vastus medialis oblique muscle in straight leg raise exercise with differ-ent angles of hip rotation. Physiotherapy. 2003; 89(7):423-30. [DOI:10.1016/S0031-9406(05)60076-4]
[34] Karst GM, Jewett PD. Electromyographic analysis of exercises proposed for differential activation of medial and lateral quadriceps femoris muscle components. Physical Therapy. 1993; 73(5):286-95. [DOI:10.1093/ptj/73.5.286]

[35] Wong Y, Ng G. Resistance training alters the sensorimotor control of vasti muscles. Journal of Electromyography and Kinesiology. 2010; 20(1):180-4. [DOI:10.1016/j.jelee kin.2009.02.006]

[36] Prins MR, Van Der Wurff P. Females with patellofemoral pain syndrome have weak hip muscles: A systematic review. Australian Journal of Physiotherapy. 2009; 55(1):9-15. [DOI:10.1016/S0004-9514(09)70055-8] 
